\title{
An Improved Ionized Impurity Scattering Model for Monte Carlo Calculations
}

\author{
G. KAIBLINGER-GRUJIN* and H. KOSINA \\ Institute for Microelectronics, TU Vienna, Gusshausstrasse 27-29, A-1040 Vienna, Austria
}

\begin{abstract}
The well known Brooks-Herring (BH) formula for charged-impurity $(\mathrm{CI})$ scattering overestimates the mobility of electrons in highly doped semiconductors. The $\mathrm{BH}$ approach relies on a static, single-site description of the carrier-impurity interactions neglecting many-particle effects. We propose a physically based charged-impurity scattering model including FermiDirac statistics, dispersive screening, and two-ion scattering. An approximation for the dielectric function is made to avoid numerical integrations. The resulting scattering rate formulas are analytical. Monte Carlo calculations were performed for majority electrons in bulk silicon at $300 \mathrm{~K}$ with impurity concentrations from $10^{15} \mathrm{~cm}^{-3}$ to $10^{21} \mathrm{~cm}^{-3}$.
\end{abstract}

Keywords: electron mobility, screening, impurity scattering, multiple scattering, Monte Carlo method, semiconductor

\section{INTRODUCTION}

Following the review by Chattopadhyay and Queisser [1], the most important causes for the failure of the BH [2] approach are: (1) screening is obviously overestimated, and (2) multiple scattering is completely ignored. Screening is a dynamic process, and the full dielectric function, which depends on the momentum transfer $q$ and the frequency $\omega$, has to be included to describe correctly the dielectric response of charged carriers to external (or internal) potentials. An early discussion of dynamic screening for non-degenerate material was given by Takimoto who takes into account the correlation effects among the conduction electrons [3]. The crucial integral expression, which actually is a simplified expression of the dielectric function, was approximated by a unit step function which conbines the $\mathrm{BH}$ and the Conwell-Weisskopf
(CW) approach. Ridley [4] considered dynamic screening by assuming only back-scattering processes $(F=0.5)$. Hall [5] used a Taylor series expansion of Takimoto's integral including terms up to fourth order. Chung and Ferry [6] developed an even more complicated integral expression suitable for arbitrary degenerate material.

Multiple scattering was taken into account by Moore [7] using a self-energy approach. Gerlach and Rautenberg showed that the interaction of the impurities cannot be neglected for concentrations larger than $5 \cdot 10^{16} \mathrm{~cm}^{-3}[8]$.

\section{PHYSICAL MODEL}

To obtain the total scattering rate $\lambda(k)$ needed in Monte Carlo calculations we first have to compute the

* Corresponding author. Tel: +43 1 58801-3851.Fax: +43 1 5059224. E-mail: Kaibling@iue.tuwien.ac.at 
differential scattering rate $W\left(\vec{k}, \overrightarrow{k^{\prime}}\right)$ from the initial state $|\vec{k}\rangle$ to the final state $\left|\overrightarrow{k^{\prime}}\right\rangle$ which is given by Fermi's golden rule :

$$
W\left(\vec{k}, \vec{k}^{\prime}\right)=\frac{N_{i}}{\hbar}\left(\frac{Z e^{2}}{2 \pi \varepsilon_{0} \varepsilon_{\tau}}\right)^{2} \frac{\partial\left(E_{\vec{k}}-E_{\vec{k}^{\prime}}\right)}{q^{4}}\left(\frac{1}{\varepsilon(q, \omega)}\right)^{2}
$$

Integration over the $\vec{k}$-space gives the total scattering rate

$$
\lambda(k)=\frac{1}{2 \pi}\left(\frac{Z e^{2}}{\varepsilon_{0} \varepsilon_{r}}\right)^{2} \frac{m^{*}(1+2 \alpha E)}{\hbar^{3} k} N_{i} \int_{0}^{2 k} \frac{d q}{q^{3}}\left[\frac{1}{\varepsilon(q, \omega)}\right]^{2}
$$

Eq.(2) does not account for the distance of the impurities, and assumes that only one charged impurity is involved in the scattering at a time.

Considering only the lowest order screening effects (linear response approach or random phase approximation) one gets a specific form of the dielectric function, the so-called Lindhard function.

$$
\varepsilon(\vec{q}, 0)=1+\frac{\beta_{s}^{2}}{q^{2}} \cdot F(\xi, \mu)
$$

with

$$
\xi=\frac{\hbar^{2} q^{2}}{8 m^{*} k_{B} T}, \quad \mu=\frac{E_{F}}{k_{B} T}, \quad \beta_{s}^{2}=\frac{n Z e^{2}}{\varepsilon_{0} \varepsilon_{r} k_{B} T}
$$

$F(\xi, \mu)[6]$ is an unsolvable integral which has to be approximated for Monte Carlo purposes. In contrast with rather rough approximations in the past (cf. ref. [3]-[5]), which are only useful for small momentum transfers, we use an adapted Lorentz function of second order

$$
F(\xi, \mu) \approx \frac{F_{-\frac{1}{2}}(\mu)}{F_{\frac{1}{2}}(\mu)} \cdot \frac{1}{1+\frac{F_{-\frac{1}{2}}(\mu)}{F_{\frac{1}{2}}(\mu)} \cdot \frac{\xi^{2}}{d}}
$$

where $F_{j}$ is the Fermi integral of order $j$. This function shows the same behavior as the integral not only for small $\xi(d=1.5$, cf. Hall [5] for non-degenerate and $d=3.75$ for strong degenerate semiconductors), but also for large $\xi(d=0.5)$ for arbitrary degeneracy. As doping increases, the average distance between two impurities becomes smaller and the neighboring ion potentials overlap appreciably, such that the singlesite-model for ionized impurity scattering breaks down. Therefore it is necessary to consider scattering processes at two ion potentials simultaneously. Equally charged pairs of impurities scatter up to twice as effectively as monopoles [9].

The total scattering rate for equally charged pairs of impurities is

$$
\begin{aligned}
& \lambda_{\text {two-ion }}(k)=\frac{1}{2 \pi}\left(\frac{Z e^{2}}{\varepsilon_{0} \varepsilon_{r}}\right)^{2} \frac{m^{*}(1+2 \alpha E)}{\hbar^{3} k} \\
& N_{i} \int_{0}^{2 k} \frac{d q}{q^{3}}\left[\frac{1}{\varepsilon(q, \omega)}\right]^{2} \cdot\left(1+\frac{\sin (q \cdot R)}{q \cdot R}\right)
\end{aligned}
$$

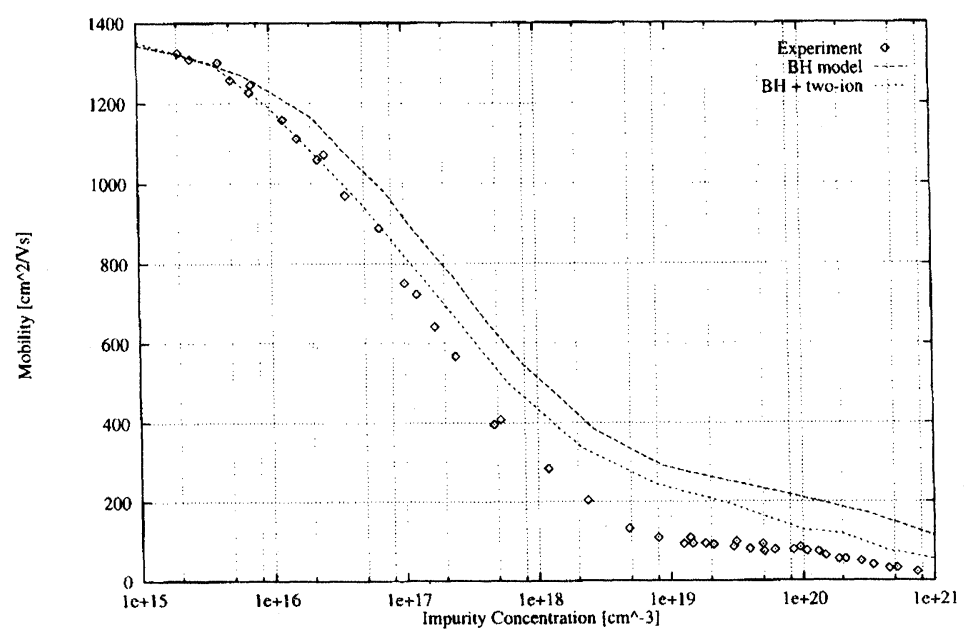

FIGURE $1 \mathrm{BH}$ model combined with the two-ion correction 
Majority electron mobilities as a function of ionized impurity concentration for silicon at $300 \mathrm{~K}$ are shown in Figs.1-3. Fig. 1 shows the significantly better agreement with experimental data $[10]$ when combining the simple BH model with the two-ion correction. It can be seen in Fig. 2 that dynamic screening becomes significant at impurity concentrations of about $10^{18} \mathrm{~m}^{-3}$. Using $d$ from 1.5 at low doping to 3.75 at high doping takes into account that with increasing degeneracy the dependence of $F(\xi \mu)$ on the momentum transfer decreases. Fig. 3 shows the results including dynamic screening $(d=0.5)$ and the two-ion correction.

The new impurity scattering model improves the agreement between theory and experimental data significantly. It is therefore more suitable for Monte Carlo calculations than the classical BH model.

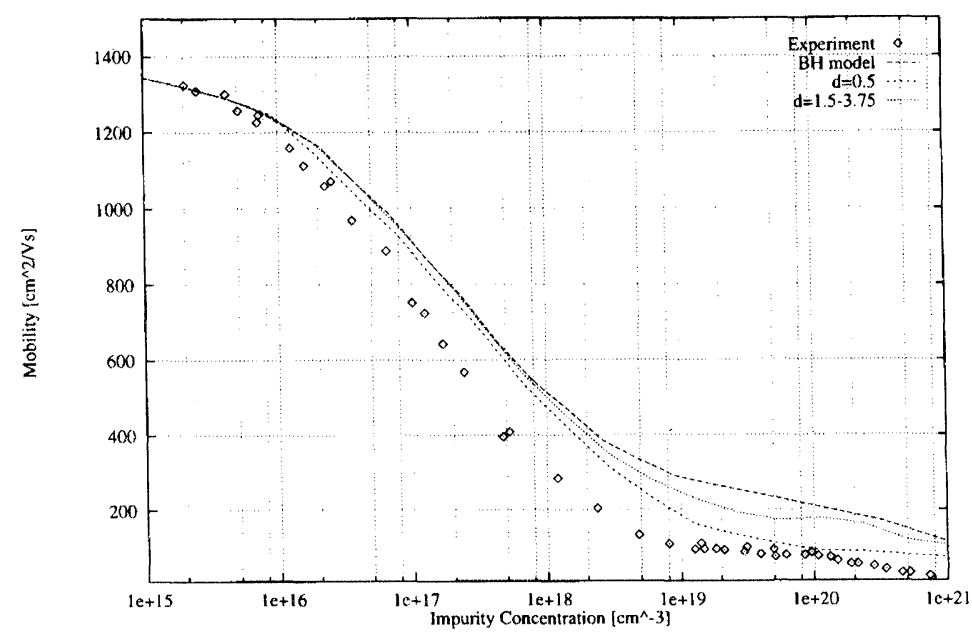

FIGURE 2 Dynamic screening correction as a function of the shape parameter $d$

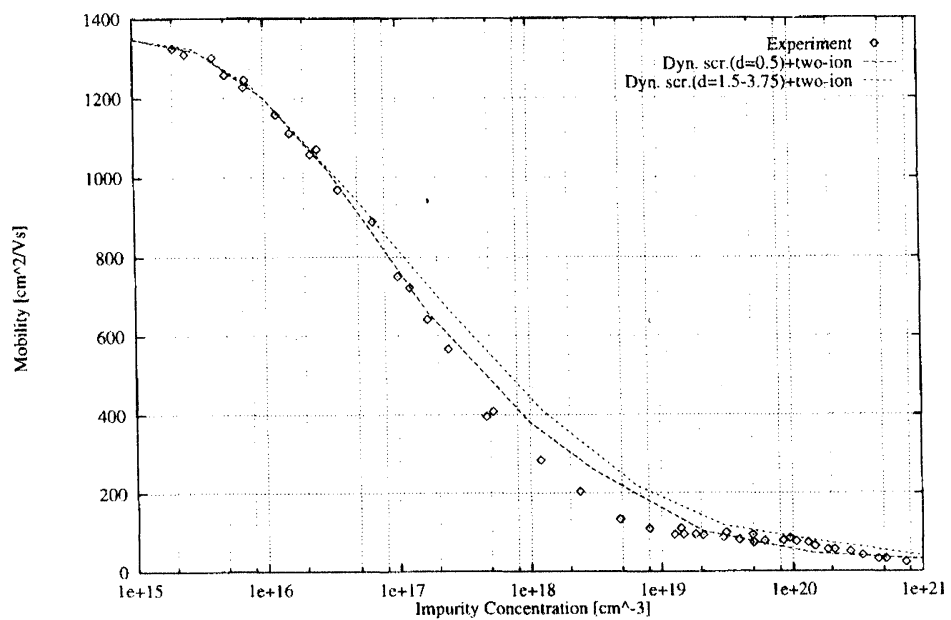

FIGURE 3 Comparison of the final results including dynamic screening and the two-ion correction with experimental data 


\section{References}

[1] D. Chattopadhyay and H. Queisser, "Electron Scattering by Ionized Impurities in Semiconductors," Rev.Mod.Phys., vol. 53, no. 4, p. 745, 1981.

[2] H. Brooks, "Scattering by Ionized Impurities in Semiconductors," Phys. Rev., vol. 83, p. 879, 1951.

[3] N. Takimoto, "On the Screening of Impurity Potential by Conduction Electrons," J.Phys.Soc.Jpn., vol. 14, no. 9, pp. $1142-1158,1959$.

[4] B. Ridley, "Charged-Impurity Scattering in GaInAs FETs," Solid-State Electron., vol. 34, no. 2, pp. 111-116, 1991.

[5] G.L. Hall, "Ionized Impurity Scattering in Semiconductors," J.Phys.Chem.Solids, vol. 23, pp. 1147-1151, 1962.

[6] W.-Y. Chung and D. Ferry, "Dynamic Screening for Ionized Impurity Scattering in Degenerate Semiconductors," SolidState Electron., vol. 31, no. 9, pp. 1369-1374, 1988.

[7] E. Moore, "Quantum-Transport Theories and Multiple Scattering in Doped Semiconductors. II. Mobility of n-type Gallium Arsenide," Phys. Rev., vol. 160, no. 3, pp. 618-626, 1967.
[8] E. Gerlach and M. Rautenberg, "Ionized Impurity Scattering in Semiconductors," Phys.stat.sol.(b), no. 86, pp. 479-482, 1978.

[9] J. Meyer and F. Bartoli, "Effect of coherent multi-ion interference on ionized-impurity scattering in semiconductors," Phys. Rev. B, vol. 30, no. 2, pp. 1026-1029, 1983.

[10] G. Masetti, M. Severi, and S. Solmi, "Modeling of Carrier Mobility Against Carrier Concentration in Arsenic-, Phosphorus- and Boron-Doped Silicon," IEEE Trans.Electron Devices, vol. ED-30, no. 7, pp. 764-769, 1983.

\section{Biographies}

Goran Kaiblinger-Grujin is currently working for his doctoral degree. His scientific interests include semiconductor physics and Monte-Carlo methods for device modeling.

H. Kosina, for biography, see 'A Hot-Hole Transport Model Based on Spherical Harmonics Expansion of the Anisotropic Bandstructure' in this issue. 

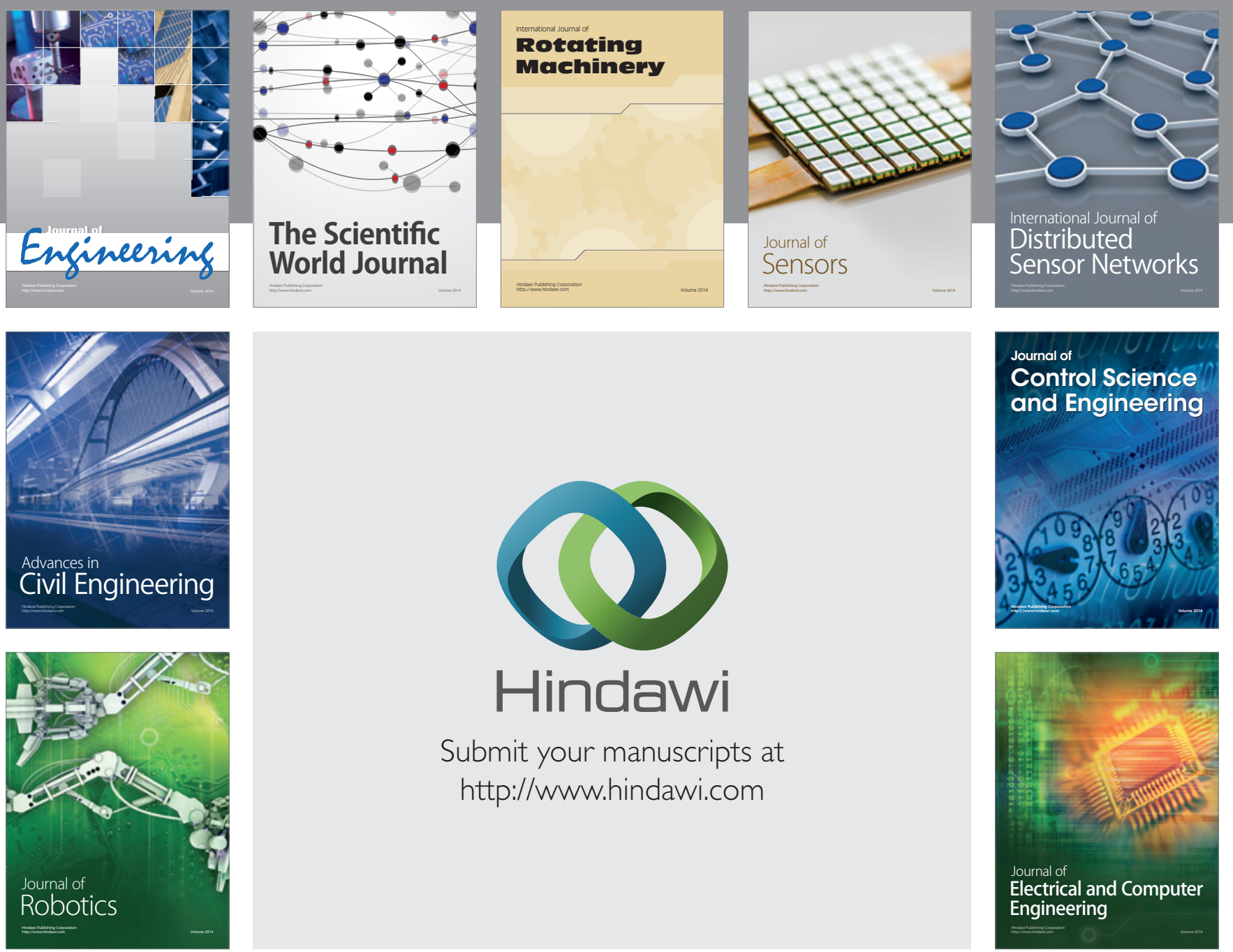

Submit your manuscripts at

http://www.hindawi.com
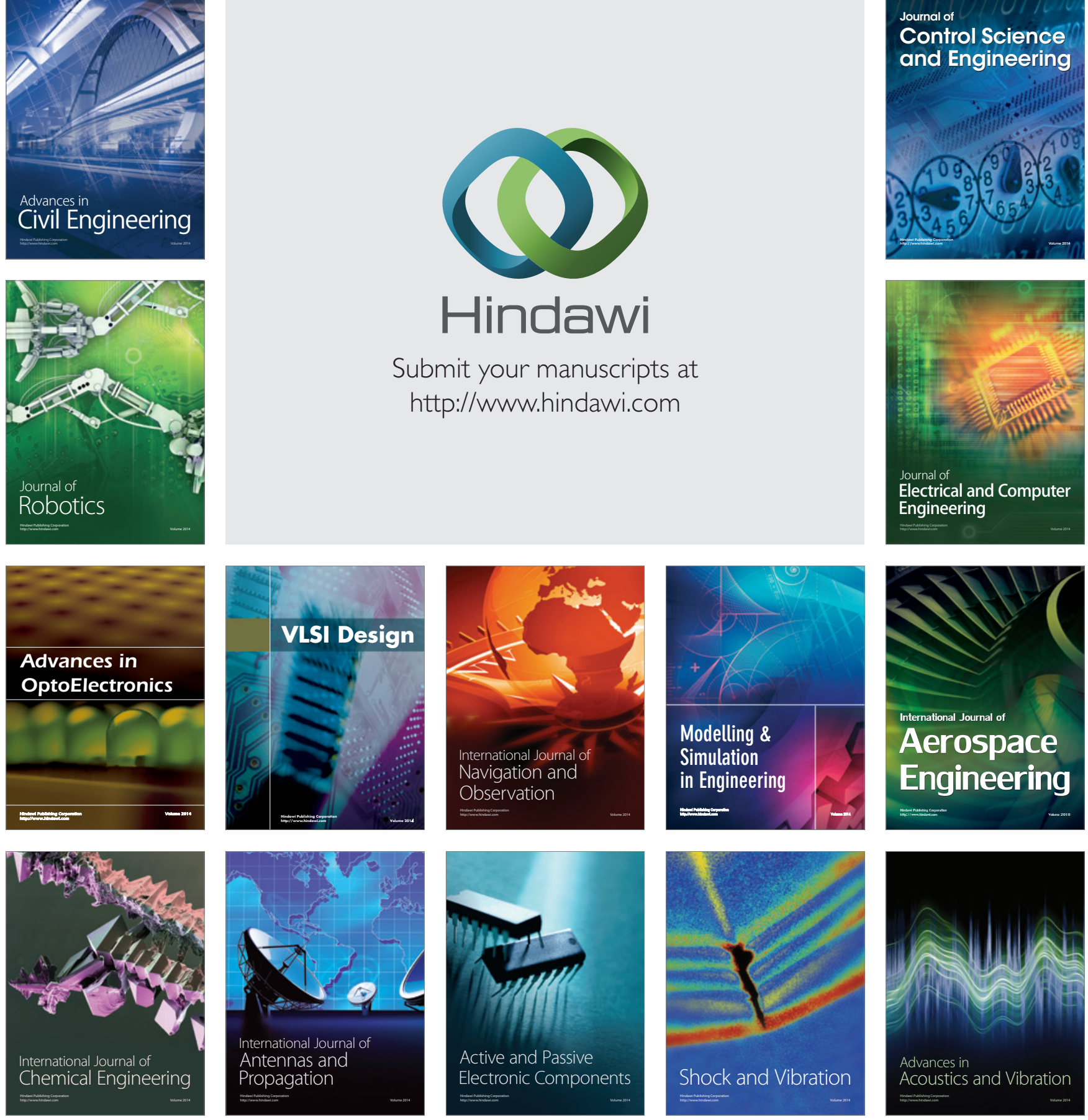